\title{
Population genetics of hyperphenylalaninaemia resulting from phenylalanine hydroxylase deficiency in Portugal
}

\author{
Isabel Rivera, Paula Leandro, Uta Lichter-Konecki, Isabel Tavares de Almeida, \\ Maria Celeste Lechner
}

\begin{abstract}
In order to elucidate the molecular basis of phenylketonuria (PKU) in Portugal, a detailed study of the Portuguese mutant phenylalanine hydroxylase (PAH) genes was performed. A total of 222 mutant alleles from 111 PKU families were analysed for 26 mutations and restriction fragment length polymorphism/variable number tandem repeat (RFLP/VNTR) haplotypes. It was possible to characterise $55 \%$ of the mutant alleles, in which 14 different mutations (R261Q, V388M, IVS10nt-11, I65T, P281L， R252W, R158Q, L348V, Y414C, L311P, Y198fsdel22bp, R408W, $R 270 K$, and $R 261 X)$ and three polymorphisms (Q232Q, V245V, and L385L) were identified. A total of 14 different haplotypes were observed, with a high prevalence of haplotype 1 among mutant and normal alleles. The results reported in this study show considerable genetic heterogeneity in the Portuguese PKU population, as has also been described for other southern European populations.

(f Med Genet 1998;35:301-304)
\end{abstract}

Keywords: Portuguese; hyperphenylalaninaemia; haplotypes; mutations

Mutations in the phenylalanine hydroxylase (PAH; EC 1.14.16.1) gene are the molecular basis of phenylketonuria (PKU; McKusick MIM 261600), an autosomal recessive disorder which, in white populations, affects 1 in 10000 livebirths. At present, far more than 300 different mutant alleles have been described at the PAH locus, ${ }^{1}$ which cause different levels of reduction in the catalytic activity of the enzyme, generating a wide spectrum of clinical and biochemical phenotypes (genotypic and phenotypic heterogeneity). Data on the distribution and relative frequencies of the mutations affecting the PAH gene product have been described for various populations, ${ }^{2-5}$ and show marked interpopulation differences in mutational spectra. It has also been observed that some associations between mutations and haplotypes are population specific, ${ }^{6}$ which, when combined with the characterisation of the $\mathrm{VNTR}^{7}$ and $\mathrm{STR}^{8}$ alleles of the PAH gene, enable the formulation of hypotheses regarding the origin and spread of PKU mutations.

In Portugal, a neonatal screening programme for hyperphenylalaninaemia (HPA) was initiated in 1979 and has by now covered 1576869 newborns, 131 of whom were found to have classical PKU, corresponding to a frequency of $1 / 12037$.

In order to characterise the Portuguese PKU population at a molecular level, we started our analysis with screening for phenylalanine hydroxylase mutations by sequence analysis of exons 7 and 11 , known to correspond to regions of the $\mathrm{PAH}$ gene containing a high number of mutations. Preliminary results ${ }^{9}$ showed the presence of several mutations described in European populations (R252W, ${ }^{10}$ $\mathrm{R} 261 \mathrm{Q},{ }^{10} \mathrm{P} 281 \mathrm{~L},{ }^{11}{ }^{12}$ IVS10nt-1 $1^{13}{ }^{14}$ ), as well as two novel mutations, R270K and V388M.

In this study, we have extended the genetic analysis to PKU patients in all of Portugal in order to define the mutational spectrum of phenylalanine hydroxylase deficiencies in this country.

\section{Materials and methods}

\section{PATIENTS AND FAMILIES}

The present study included 111 hyperphenylalaninaemic children, born to unrelated, healthy parents, who originated from north Portugal (67) or south/central Portugal (48). Genomic DNA of the first group of patients was obtained from Guthrie cards, while that from the patients, and in some cases their families, living in the southern/central regions of the country was extracted from peripheral blood leucocytes.

The patients were classified, according to their initial phenylalanine levels, as having classical PKU (69 cases), mild PKU (35 cases), or non-PKU hyperphenylalaninaemia (seven cases).

Most of the patients (93 cases) were detected by the neonatal screening programme and, in every case, hyperphenylalaninaemia resulting from PAH deficiency was assessed after exclusion of tetrahydrobiopterin defiencies. The other 18 patients were born before 1980 and were clinically classified as having classical PKU. Two normal and two mutant alleles were accounted for per family. All of the 111 patients were white.

\section{HAPLOTYPE ANALYSIS}

The Southern blotting technique was used for the detection of the EcoRI and EcoRV polymorphisms; the full length cDNA probe phPAH $247^{15}$ was labelled with $\left[{ }^{32} \mathrm{P}\right] \mathrm{dCTP}$ (Amersham, UK) using the random priming method (Pharmacia Biotech, Uppsala, Swe- 
Table 1 Mutations and polymorphisms screened in the Portuguese HPA population by restriction digestion

\begin{tabular}{ll}
\hline G46S & $(-)$ Sau96I (ACRS) \\
L48S & $(+)$ SfaNI \\
I65T & $(-)$ TaqI (ACRS) \\
R158Q & $(-)$ MspI (ACRS) \\
Y198fsdel22 & Gel electrophoresis \\
Q232Q & $(+)$ DdeI \\
V245A & $(+)$ Fnu4HI \\
V245V & $(+)$ AluI \\
R252W & $(-)$ AvaI \\
R261Q & $(-)$ HinfI \\
R261X & $(+)$ DdeI \\
G272X & $(-)$ BamHI \\
E280K & $(-)$ MspI (ACRS) \\
P281L & $(-)$ Sau96I (ACRS) \\
IVS7nt1 & $(+)$ NlaIII \\
F299C & $(-)$ HindIII (ACRS) \\
L311P & $(+)$ MspI \\
L348V & $(-)$ NlaIV \\
IVS10nt-11 & $(+)$ DdeI \\
L385L & $(+)$ MnII \\
V388M & $(-)$ BsaAI \\
A403V & $(-)$ BbvI \\
R408Q & $(-)$ Sau96I \\
R408W & $(-)$ MnII / (+) StyI \\
Y414C & $(+)$ BbvI / (-) RsaI (ACRS) \\
IVS12nt1 & $(-)$ RsaI (ACRS) \\
\hline
\end{tabular}

ACRS - amplification created restriction site; $(+)$ site created $(-)$ site delected.

den), and hybridised under conditions described elsewhere. ${ }^{16}$

The intragenic RFLPs at BglII, PvuIIa, $P v u I I b, M s p I$, and $X m n I$ sites were analysed by polymerase chain reaction (PCR) amplification, as described previously. ${ }^{17-20}$ Analysis of the VNTR system at the HindIII site at the 3 ' end of the gene was performed according to Goltsov et al. ${ }^{7}$

Haplotypes were numbered in accordance with the nomenclature established by $\mathbf{W o o}^{21}$ and updated by Eisensmith and Woo. ${ }^{22}$

\section{MUTATION ANALYSIS}

Ten different exons and flanking intronic regions were amplified by PCR, as previously reported. ${ }^{9}$ Known mutations were screened by a restriction enzyme based assay, using naturally occurring or amplification created sites ${ }^{23}$ (table 1), and in some cases by simultaneous allele specific oligonucleotide hybridisation (ASO). Screening for unknown mutations was performed by sequence analysis using the dideoxy chain termination method. ${ }^{24}$

Enzymatic digestion of PCR products

Twenty three mutations and three polymorphisms were screened for by restriction digestion of PCR products with the appropriate enzymes (table 1), followed by electrophoretic separation on Nusieve GTG agarose (FMC).

Allele specific oligonucleotide hybridisation ASO screening was performed on PCR products adsorbed to nylon membranes (Boehringer Mannheim, Germany) and analysed separately, for the normal and the mutant alleles, with digoxigenin labelled probes and chemiluminescent detection with $\operatorname{CSPD}^{k}$ (Boehringer Mannheim, Germany).

\section{Results}

MUTATION ANALYSIS

Screening for 23 mutations and three polymorphisms (table 1), previously described in other white populations, allowed the identification of the three polymorphisms and 14 of these mutations in the Portuguese population. Table 2 shows that $55 \%(122 / 222)$ of all PKU alleles studied were found to carry a previously identified mutation and that there is no one mutation(s) prevalent in the Portuguese PKU population; rather three groups of mutations exist for which relative frequencies are $9-11 \%$ (IVS10nt-11, R261Q, V388M), 3-6\% (I65T, P281L, R252W, R158Q), and below 2\% (L348V, Y414C, L311P, Y198fsdel22, R408W, R270K, R261X), respectively.

Previously described diallelic polymorphisms were identified in the Portuguese population in codons 232,245 , and 385 . The frequencies of these polymorphisms were found to be $10.4 \%$ for the $G$ allele and $89.6 \%$ for the A allele of the $\mathrm{Q} 232 \mathrm{Q}$ polymorphism, $9.4 \%$ for the $A$ allele and $90.6 \%$ for the $G$ allele of the $\mathrm{V} 245 \mathrm{~V}$ polymorphism, and $3.2 \%$ for the $C$ allele and $96.8 \%$ for the $G$ allele of the L385L polymorphism.

Determination of the complete genotype was possible in 41 of the 111 patients $(37 \%)$; in an additional 40 patients one of the mutant alleles could be identified. In the group of the fully genotyped patients, $44 \%$ (18/41) were homozygous for one mutation while the remaining 23 patients were compound heterozygotes for two mutations. The homozygosity rate in the Portuguese population is $22.5 \%$, similar to the average European rate $(-25 \%)$.

In those patients in whom it was impossible to detect both mutations, sequence analysis was performed to search for those exons harbouring mutations associated with the haplotype of the patient. Sequence analysis of 10 exons (exons 2, 3, and 5 to 12) of the PAH gene allowed the identification of two novel mutations, $\mathrm{R} 270 \mathrm{~K}$ and V388M. The latter could be subsequently screened for by restriction enzyme analysis with $B s a \mathrm{AI}$ (table 1).

\section{HAPLOTYPE ANALYSIS}

Unambiguous characterisation of the haplotypes was possible in 24 families living in south/central Portugal, corresponding to 48 mutant and 40 normal alleles. The difference between the number of mutant and normal alleles is because, in some cases, only the DNA of one parent was available; however, in these cases the mutant alleles could still be determined because the patients were homozygous.

Fourteen different haplotypes were identified, with the HindIII VNTR polymorphic site displaying heterogeneity of haplotypes $1,5,7$, and 36 alleles (table 3 ). It is interesting to note the high prevalence of haplotype 1 , among normal $(27.5 \%)$ and especially mutant chromosomes $(64.6 \%)$. Overall, the most frequent haplotypes in the Portuguese population are common among normal as well as mutant alleles, except for haplotype 6 which is exclusively associated with mutant chromosomes in the patient population tested.

\section{MUTATION-HAPLOTYPE ASSOCIATION}

A correlation between mutations and haplotypes could only be assessed for southern Portuguese mutant alleles since haplotype 
Table 2 Distribution of HPA causing mutations in 222 unrelated Portuguese PKU chromosomes

\begin{tabular}{lccl}
\hline Mutation & $\begin{array}{l}\text { No of positive } \\
\text { chromosomes }\end{array}$ & Relative frequency (\%) & $\begin{array}{l}\text { RFLPVNTR } \\
\text { haplotype }\end{array}$ \\
\hline IVS10nt-11 & 24 & 10.8 & 6.7 \\
R261Q & 23 & 10.4 & 1.8 \\
V388M & 19 & 8.6 & 1.7 \\
I65T & 12 & 5.4 & $9.8 / 57.8$ \\
P281L & 10 & 4.5 & 1.8 \\
R252W & 9 & 4.0 & 1.8 \\
R158Q & 7 & 3.2 & 4.3 \\
L348V & 4 & 1.8 & 9.8 \\
Y414C & 4 & 1.8 & 4.3 \\
L311P & 3 & 1.4 & 3.8 \\
Y198fsdel22 & 2 & 0.9 & 5.9 \\
R408W & 2 & 0.9 & $1.8 / 2.3$ \\
R270K & 2 & 0.9 & 1.8 \\
R261X & 1 & 0.4 & 4.3 \\
Uncharacterised & 100 & 45.0 & \\
Total & 222 & 100.0 & \\
\hline
\end{tabular}

Table 3 Polymorphic haplotypes identified on normal and mutant chromosomes in 24 unrelated Portuguese PKU families

\begin{tabular}{|c|c|c|c|c|}
\hline \multirow[b]{2}{*}{ Haplotype.VNTR } & \multicolumn{2}{|c|}{ Normal alleles } & \multicolumn{2}{|c|}{ Mutant alleles } \\
\hline & No & $\%$ & No & $\%$ \\
\hline $1.7+1.8$ & $11(3+8)$ & 27.5 & $31(10+21)$ & 64.6 \\
\hline 3.8 & 1 & 2.5 & 1 & 2.1 \\
\hline 4.3 & 3 & 7.5 & 4 & 8.3 \\
\hline $5.7+5.9$ & $5(1+4)$ & 12.5 & $2(0+2)$ & 4.2 \\
\hline 6.7 & - & - & 6 & 12.5 \\
\hline $7.7+7.8$ & $6(1+5)$ & 15.0 & $1(0+1)$ & 2.1 \\
\hline 27.9 & 2 & 5.0 & - & - \\
\hline 28.3 & 3 & 7.5 & - & - \\
\hline 31.3 & 3 & 7.5 & - & - \\
\hline 34.8 & 1 & 2.5 & - & - \\
\hline $36.8+36.9$ & $2(0+2)$ & 5.0 & $3(2+1)$ & 6.2 \\
\hline 41.3 & 1 & 2.5 & - & - \\
\hline 52.8 & 1 & 2.5 & - & - \\
\hline 57.8 & 1 & 2.5 & - & - \\
\hline Total & 40 & 100.0 & 48 & 100.0 \\
\hline
\end{tabular}

analysis was not possible in northern Portuguese families.

Each of the mutations found in the Portuguese PKU population was strongly associated with a single RFLP/VNTR haplotype, except for the R408W and I65T mutations. The first mutation was observed on the background of haplotypes 1.8 and 2.3 (once in both cases), while the I65T mutation was found in association with haplotype 9.8 and once with haplotype 57.8. Interestingly, I65T was associated with the same VNTR allele in both haplotypes.

Six mutations (R252W, R261Q, R270K, P281L, V388M, R408W) were detected associated with haplotype 1 alleles, which comprise $64.6 \%$ of the mutant alleles in the Portuguese population, three mutations associated with haplotype 4 (R158Q, R261X, Y414C) and two mutations on the background of haplotype 9 (I65T, L348V) alleles.

As in other populations, the diallelic polymorphisms detected in our sample were strongly associated with specific haplotypes: Q232Q with haplotypes 3, 4, and 7; V245V exclusively with haplotype 4 ; and L385L with haplotypes 3 and 7 .

\section{Discussion}

The results obtained in the present study confirm the considerable genetic heterogeneity of the Portuguese PKU population, a situation already described for other southern European populations. ${ }^{25-27}$ This heterogeneity might re- flect the large number of different populations who have inhabited this western part of the Iberian Peninsula, which later on became Portugal.

Though haplotype 1 is present in both mutant and normal alleles throughout Europe, the frequency of the mutant haplotype 1 alleles varies in different regions. The results obtained in the present study show that mutant haplotype 1, with a relative frequency of $64.6 \%$, is very prevalent among PKU alleles in Portugal, as was observed before. ${ }^{28}$ Nevertheless, no clearly prevalent mutation was determined to be associated with haplotype 1 alleles in Portugal in this study.

The 14 mutations identified in our study were all found on the background of the haplotypes previously described as being associated with them. Therefore, previous hypotheses about the origin of these mutations may also apply to the Portuguese population. The I65T mutation was found on haplotytpe 9.8 and only once on haplotype 57.8 , a rare haplotype, which could be derived from haplotype 9 by a mutation altering the $E c o$ RI site in the PAH gene. Interestingly, a normal haplotype 57 allele was also detected in the Portuguese PKU population by Caillaud et al. ${ }^{28}$

The results obtained in the present study show that the most frequent mutation observed in the Portuguese PKU population is IVS10nt11 , with a relative frequency of $11.1 \%$, as has been reported for every southern European population studied. ${ }^{25-27}$ 29-31 A possible Middle Eastern origin of this mutation has been discussed previously ${ }^{27}$ and, in agreement with this hypothesis, one could speculate that this mutation might have been brought to Portugal by the Phoenicians, who established many trading points along the Portuguese coast.

In Portugal, as in Sicily, ${ }^{25}$ the R261Q mutation is the second most frequent mutation. Interestingly, the R261Q mutation presents with a high relative frequency in Switzerland, where its postulated origin is, ${ }^{32}$ but its incidence is very low in Spain, ${ }^{26}$ which is not in agreement with a north/south decline of this mutation frequency.

The V388M mutation, described for the first time in white populations by our group, ${ }^{9}$ is the third most frequent mutation in Portugal; it has also been found at a similarly high frequency $(8.1 \%)$ in Spain. ${ }^{26}$ In both countries this mutation is linked to haplotype 1.7. This same mutation has also been detected in Latin American populations, ${ }^{33} 34$ namely in Brazil and Chile, which are former colonies of Portugal and Spain, respectively. In view of this fact, together with its virtual absence from other European populations, ${ }^{25}$ the V388M mutation on the background of haplotype 1.7 may be regarded as an Iberian mutation, rather than a Spanish one, as was postulated by Desviat $e t$ al. $^{33}$

Recently, a detailed study of the PAH mutation profile of the Spanish population has been published. ${ }^{35}$ Comparing the Portuguese and the Spanish mutational spectra, it is obvious that the four major mutations are common to both populations and that they are found on 
the background of the same main haplotypes (IVS10nt-11/H6.7; R261Q/H1.8; V388M/ $\mathrm{H} 1.7$; I65T/H9.8). Regarding the other mutations present in both populations, their incidence is markedly different and, interestingly, most of them are in association with different haplotypes; this is the case with the L $311 \mathrm{P}$ and the R252W mutations, associated with haplotypes 3.8 and 1.8, and haplotypes 17.9/7.9 and X.3, respectively, in Portugal and in Spain. There are also five mutations present in the Portuguese population that are completely absent from the Spanish sample, particularly the $\mathrm{R} 158 \mathrm{Q}$ and the $\mathrm{L} 348 \mathrm{~V}$ mutations, found at a relatively high frequency in Portugal.

Of particular interest is the observed absence of the A403V mutation in the Portuguese HPA patients, because this mutation is described as the most frequent one among southern European non-PKU HPA patients. ${ }^{25}{ }^{36}$ Until now, none of the already described non-PKU mutations has been identified in our population.

The present information regarding the Portuguese HPA population, together with the above mentioned study on the mutation profile in Spain, seems to prove that both populations, though very closely situated, have received distinct ethnic influences throughout history.

In conclusion, the present study provides data for a population genetic analysis of the distribution and spread of PKU mutations in southern Europe, where Portugal assumes a unique position with regard to its composition of PKU alleles.

The authors wish to thank Dr Savio L C Woo for supplying the phPAH247 cDNA probe and Dr Hans-Geir Eiken for having kindly provided many of the ACRS primers for PCR. This work was supported by a grant from the Junta Nacional de Investigação Científica e Tecnológica (SAU 34/95).

1 Nowacki P, Byck S, Prevost L, Scriver CR. The PAH mutation analysis consortium database: update 1996. Nucleic Acids Res 1997;25:139-42.

2 Guldberg P, Henriksen KF, Güttler F. Molecular analysis of phenylketonuria in Denmark: $99 \%$ of the mutations detected by denaturing gradient gel electrophoresis. detected by denaturing
Genomics 1993;17:141-6.

3 Kleiman S, Avigad S, Vanagaite L, et al. Origins of Kleiman S, Avigad S, Vanagaite $\mathrm{L}$, et al. Origins of hyperp.

4 Okano Y, Hase Y, Lee DH, et al. Molecular and population genetics of phenylketonuria in Orientals. $\mathcal{F}$ Inherit Metab Dis 1994;17:156-9.

5 Rozen R, Mascish A, Lambert M, Laframboise R, Scrive CR. Mutation profiles of phenylketonuria in Quebec populations: evidence of stratification and novel mutations. Am $\mathcal{F}$ Hum Genet 1994;55:321-6.

6 Scriver CR, John SMW, Rozen R, Eisensmith RC, Woo SLC. Associations between population, phenylketonuria mutations and RFLP haplotypes at the phenylalanine hydroxylase gene: an overview. Dev Brain Dysfunct 1993;6: 11-25.

7 Goltsov AA, Eisensmith RC, Konecki DS, Lichter-Konecki U, Woo SLC. Association between mutations and a VNTR in woo SLC. Association between mutations and a VNTR in the human phenylalani

8 Goltsov AA, Eisensmith RC, Naughten ER, et al. A single Goltsov AA, Eisensmith RC, Naughten ER, et al. A single
polymorphic STR system in the human phenylalanine polymorphic STR system in the human phenylalanine hydroxylase gene permits rapid prenatal diagnosis and car-
rier screening for phenylketonuria. Hum Mol Genet 1993;2 rier scre $577-81$.

9 Leandro P, Rivera I, Ribeiro V, Tavares de Almeida I, Silveira C, Lechner MC. Mutation analysis of phenylke- tonuria in south and central Portugal: prevalence of V388M mutation. Hum Mutat 1995;6:192-4

10 Abadie V, Lyonnet S, Maurin N, et al. CpG dinucleotides are mutation hot spots in phenylketonuria. Genomics 1989 ; 5:936-9.

11 Okano Y, Wang T, Eisensmith RC, et al. Phenylketonuria missense mutations in the Mediterranean. Genomics 1991; 9:96-103.

12 Dworniczak B, Grudda K, Stümper J, Bartholomé K, Aulehla-Scholz C, Horst J. Phenylalanine hydroxylase gene: novel missense mutation in exon 7 causing severe phenylketonuria. Genomics 1991;9:193-9.

13 Dasovich M, Konecki D, Lichter-Konecki U, et al. Molecular characterization of PKU allele prevalent in southern Europe and Ireland. Somat Cell Mol Genet 1991;17:303-9.

14 Kalaydjieva L, Dworniczak B, Aulehla-Scholz C, et al. Phenylketonuria mutations in southern Europeans. Lancet 1991;337:865

15 Kwok SCM, Ledley FD, DiLella AG, et al. Nucleotide sequence of a full-length complementary DNA clone and amino acid sequence of human phenylalanine hydroxylase. Biochemistry 1985;24:556-61.

16 Church GM, Gilbert W. Genomic sequencing. Proc Natl Acad Sci USA 1984;81:1991-5.

17 Dworniczak B, Wedemeyer N, Horst J. PCR detection of the BglII RFLP at the human phenylalanine hydroxylase (PAH) locus. Nucleic Acids Res 1991;19:1958.

18 Dworniczak B, Wedemeyer N, Eigel A, Horst J. PCR detection of the PvulI (Ea) RFLP at the human phenylalanine hydroxylase (PAH) locus. Nucleic Acids Res 1991;19:1958.

19 Wedemeyer N, Dworniczak B, Horst J. PCR detection of the MspI (Aa) RFLP at the human phenylalanine hydroxylase (PAH) locus. Nucleic Acids Res 1991;19:1959.

20 Goltsov AA, Eisensmith RC, Woo SLC. Detection of the XmnI RFLP at the human PAH locus by PCR. Nucleic Acids Res 1991;20:927.

21 Woo SLC. Collation of RFLP haplotypes at the human phenylalanine hydroxylase PAH locus. Am ₹ Hum Genet 1988; 43:781-3

22 Eisensmith RC, Woo SLC. Update listing of haplotypes at the human phenylalanine hydroxylase (PAH) locus. $\mathrm{Am} \mathcal{F}$ Hum Genet 1992;51:1445-8.

23 Eiken HG, Knappskog PM, Apold J. Restriction enzymebased assays for complete genotyping of phenylketonuria patients. Dev Brain Dysfunct 1993;6:53-9.

24 Sanger F, Nicklen S, Caulson AR. DNA sequencing with chain-terminating inhibitors. Proc Natl Acad Sci USA 1977;75:5463-7.

25 Guldberg P, Romano V, Ceratto N, et al. Mutational spectrum of phenylalanine hydroxylase deficiency in Sicily: implications for diagnosis of hyperphenylalaninemia in implications for diagnosis of hyperphenylalanin

26 Pérez B, Desviat LR, De Lucca M, Ugarte M. Spectrum and origin of phenylketonuria mutations in Spain. Acta Paediatr Scand Suppl 1994;407:34-6.

27 Dianzani I, Giannattasio S, de Sanctis L, et al. Characterization of phenylketonuria alleles in the Italian population. Eur f Hum Genet 1995;3:294-302.

28 Caillaud C, Vilarinho L, Vilarinho A, et al. Linkage disequilibrium between phenylketonuria and RFLP haplotype 1 at the phenylalanine hydroxylase locus in Portugal. Hum the phenylalanine hydr

29 Özgüç M, Özalp I, Coskun T, Yilmaz E, Erdem H, Ayter S. Mutation analysis in Turkish phenylketonuria patients. $f$ Med Genet 1993;30:129-30.

30 Traeger-Synodinos J, Kanavakis E, Kalogerakou M, Soulpi $\mathrm{K}$, Missiou-Tsangaraki S, Kattamis C. Preliminary mutation analysis in the phenylalanine hydroxylase gene in Greek PKU and HPA patients. Hum Genet 1994;94:573-5.

31 Hashem N, Bosco P, Chiavetta V, Cali F, Ceratto N, Romano V. Preliminary studies on the molecular basis of hyperper

Eisensmith RC, Okano Y, Dasovich M, et al. Multiple Eisensmith RC, Okano Y, Dasovich M, et al. Multiple origins for phenylket

33 Desviat LR, Peréz B, De Lucca M, et al. Evidence in Latin America of recurrence of $\mathrm{V} 388 \mathrm{M}$, a phenylketonuria mutation with high in vitro residual activity. Am f Hum Gene 1995;57:337-42.

34 Peréz B, Desviat LR, De Lucca M, et al. Mutation analysis of phenylketonuria in south Brazil. Hum Mutat 1996;8 262-4.

35 Peréz B, Desviat LR, Ugarte M. Analysis of the phenylalanine hydroxylase gene in the Spanish population: lanition profile and association with intragenic polymorphic markers. Am f Hum Genet 1997;60:95-102.

36 Desviat LR, Peréz B, Ugarte M. Molecular basis of non-PKU hyperphenylalaninaemia in Spain: prevalence of A403V, a mutation with high residual activity. $\mathcal{F}$ Inherit Metab Dis 1996;19:227-30. 